\title{
AP-VAS 2012 case report: MPO-ANCA-negative relapse of MPO-ANCA-associated vasculitis
}

\author{
Yoshinori Komagata Kazufumi Nomura $\cdot$ Ken Yoshihara \\ Soko Kawashima · Shinya Kaname • Yoshihiro Arimura • \\ Akira Yamada
}

Received: 28 July 2013/Accepted: 12 September 2013/Published online: 7 November 2013

(C) Japanese Society of Nephrology 2013

\begin{abstract}
A 79-year-old female was admitted to our hospital with fever, proteinuria, hematuria, high levels of C-reactive protein (CRP), and high titer of myeloperoxidase antineutrophil cytoplasmic antibody (MPO-ANCA). Our diagnosis was microscopic polyangiitis (MPA) and she was treated with steroid pulse therapy. Clinical remission was induced; however, the disease relapsed with saddle nose and necrotizing vasculitis of the nasal cavity mucosa 1 year later. Although there was no elevation of the MPOANCA titer, we diagnosed the patient with relapse of MPO-ANCA-positive granulomatosis with polyangiitis (GPA). Remission was induced again with steroids and azathioprine. It has been reported that the number of MPOANCA-positive patients in Asian countries is relatively higher than in Western countries. We checked 29 GPA patients in our hospital and 9 patients $(31.0 \%)$ were MPOANCA-positive. In addition, it is not rare that an ANCAassociated vasculitis (AAV) patient who has been in remission with negative ANCA relapses without any elevation of ANCA titer. We checked the transition of ANCA titer of $24 \mathrm{AAV}$ patients in our hospital who relapsed and 6 patients $(25 \%)$ relapsed without any elevation of ANCA titer. We should be careful for a relapse, even if the ANCA titer remains negative. It is also possible that ANCA had been changed so as not to be detected by the same enzymelinked immunosorbent assay (ELISA) kit. Thus, it is also important to change the detection system if clinical symptoms are worsened while ANCA is still negative.
\end{abstract}

Y. Komagata $(\bowtie) \cdot$ K. Nomura $\cdot$ K. Yoshihara .

S. Kawashima - S. Kaname - Y. Arimura · A. Yamada

First Department of Internal Medicine, Kyorin University School

of Medicine, Tokyo, Japan

e-mail: komagata@ks.kyorin-u.ac.jp
Keywords ANCA-associated vasculitis · MPOANCA-positive GPA · Relapse · ELISA

\section{Introduction}

It has been reported that the ratio of myeloperoxidase antineutrophil cytoplasmic antibody (MPO-ANCA)-positive granulomatosis with polyangiitis (GPA) to all cases of GPA is relatively higher in Asian countries [1-4]. We have often experienced that GPA patients are diagnosed as microscopic polyangiitis (MPA) because of positive MPOANCA. We report here an MPO-ANCA-positive GPA patient who was also diagnosed as MPA and relapsed with saddle nose after remission induction. She relapsed without an increase of MPO-ANCA titer, and predicting relapse is very important for the maintenance of remission. We also discuss the ANCA-negative relapse of ANCA-associated vasculitis (AAV).

\section{Case}

The patient is a 79-year-old female, who visited our hospital with fever, proteinuria, hematuria, and edematous lower extremities. She had been diagnosed with polymyalgia rheumatica by another doctor and had already been treated with $15 \mathrm{mg}$ per day of prednisolone (PSL). She was admitted to our hospital because her blood tests showed marked elevation of inflammatory markers and high titer of MPO-ANCA.

At the first visit, abnormal findings on physical examination were only slight fever and edema of legs. As for laboratory tests, however, there were high white blood cell (WBC) counts, anemia, low level of serum albumin, high 
Table 1 Laboratory tests at the first visit and at relapse

\begin{tabular}{lll}
\hline & At the first visit & At relapse \\
\hline WBC & $14000 / \mu \mathrm{l}$ & $10900 / \mu \mathrm{l}$ \\
Seg & $90.5 \%$ & $92.5 \%$ \\
Eosino & $2.0 \%$ & $1.0 \%$ \\
$\mathrm{Hb}$ & $7.4 \mathrm{~g} / \mathrm{dl}$ & $8.8 \mathrm{~g} / \mathrm{dl}$ \\
$\mathrm{Alb}$ & $1.8 \mathrm{~g} / \mathrm{dl}$ & $2.1 \mathrm{~g} / \mathrm{dl}$ \\
$\mathrm{CRP}$ & $7.2 \mathrm{mg} / \mathrm{dl}$ & $11.2 \mathrm{mg} / \mathrm{dl}$ \\
$\mathrm{RF}$ & $182 \mathrm{U} / \mathrm{ml}$ & $21 \mathrm{U} / \mathrm{ml}$ \\
MPO-ANCA (NIPRO kit) & $425 \mathrm{EU}$ & $<10 \mathrm{EU}$ \\
PR3-ANCA & $<10 \mathrm{EU}$ & $<10 \mathrm{EU}$ \\
Urine protein & $5.3 \mathrm{~g} / \mathrm{day}$ & \pm \\
Urine OB & $3+$ & \pm \\
Urine glucose & $4+$ & $3+$ \\
\hline
\end{tabular}

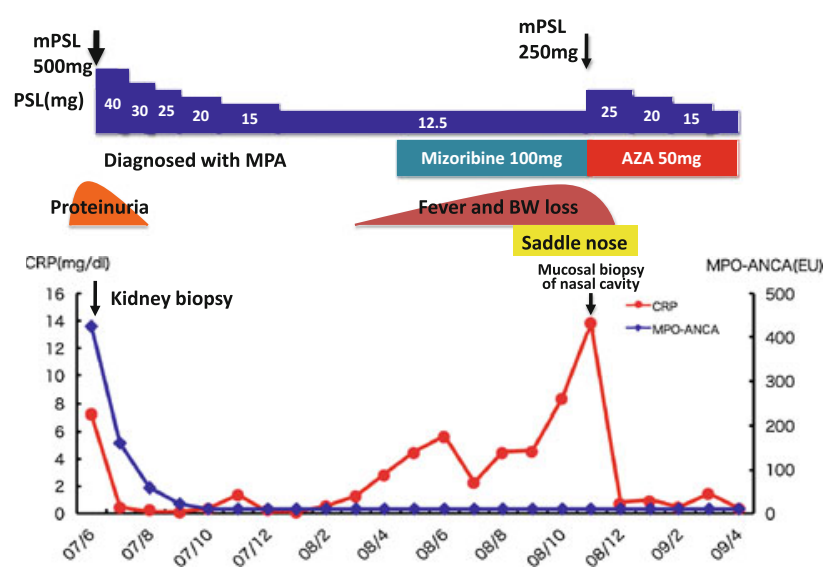

Fig. 1 Time course of the disease

blood sugar, high levels of C-reactive protein (CRP) and rheumatoid factor, and high titer of MPO-ANCA that was measured using the NIPRO enzyme-linked immunosorbent assay (ELISA) kit. PR3-ANCA was negative. In addition, urinalysis showed severe proteinuria and hematuria, although there was no renal dysfunction (Table 1). There was no eosinophilia and no history of asthma.

To confirm whether severe proteinuria can be explained by ANCA-associated glomerulonephritis, we performed a kidney biopsy. The basement membranes of most of the glomeruli were diffusely thickened. Necrotizing lesions were seen in some glomeruli and some glomeruli showed crescent formation and spike formation. Immunofluorescence (IF) microscopy revealed granular deposition of IgG along the basement membrane. Electron microscopy imaging showed some dense deposits in the basement membrane.

By kidney biopsy, we confirmed the patient had necrotizing glomerulonephritis and membranous nephropathy. Our diagnosis was MPA with membranous nephropathy at
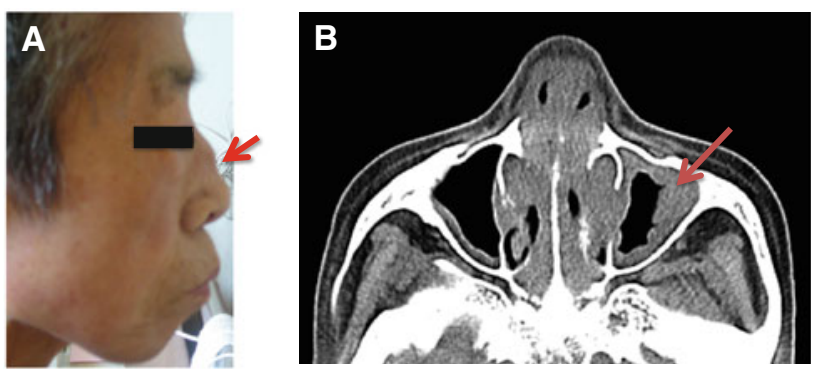

Fig. 2 Nasal symptoms at relapse. a Saddle nose. b Computed tomography (CT) scan showed a mass lesion suggestive of granuloma in the paranasal cavity with bone destruction

this point. We first tried to treat this patient with steroids. She was treated with methylprednisolone (mPSL) pulse therapy, followed by $40 \mathrm{mg}$ per day of oral PSL. Fever and proteinuria improved, and the levels of CRP and MPOANCA became within normal limits. We did not use any other immunosuppressant because the response to steroids was quite good. Clinical remission was induced 3 months after the initial therapy and the dose of PSL was tapered to $12.5 \mathrm{mg}$ per day (Fig. 1).

However, 1 year after the remission induction, the patient gradually started to suffer fever, body weight loss, and elevation of the CRP level. At the same time, we recognized that she gradually showed saddle nose, which was not seen at onset. Computed tomography (CT) scan of the paranasal cavity showed thickening of the mucosa and bone destruction (Fig. 2). Biopsy of the mucosa of the nasal cavity showed necrotizing vasculitis and leukocytoclastic vasculitis, but there was no granuloma lesion which involved giant cells (Fig. 3). The level of CRP at relapse was as high as that at onset; however, MPO-ANCA was still negative by ELISA and PR3-ANCA also remained negative, although p-ANCA by IF was weakly positive. In addition, there was no severe proteinuria or hematuria.

Based on the clinical and pathological findings since the onset of disease, we thought that she has suffered MPOANCA-positive GPA from the onset and the disease relapsed at this point. For remission induction, the patient was treated with $\mathrm{mPSL}$ pulse therapy $(250 \mathrm{mg} / \mathrm{day} \times 3)$ followed by oral PSL ( $25 \mathrm{mg} /$ day) with $50 \mathrm{mg}$ of azathioprine. Lesions in the nasal cavity improved and the level of CRP became within normal limits. Eventually, the dose of PSL was tapered to $10 \mathrm{mg} /$ day without any relapse.

\section{Discussion}

MPO-ANCA-positive GPA

It has been reported that the ratio of MPO-ANCA-positive GPA to all cases of GPA is around $20 \%$ in Western 
Fig. 3 Biopsy of the nasal cavity at relapse. a Necrotizing vasculitis (Elastica van Gieson stain). b Leukocytoclastic vasculitis (hematoxylin and eosin stain)

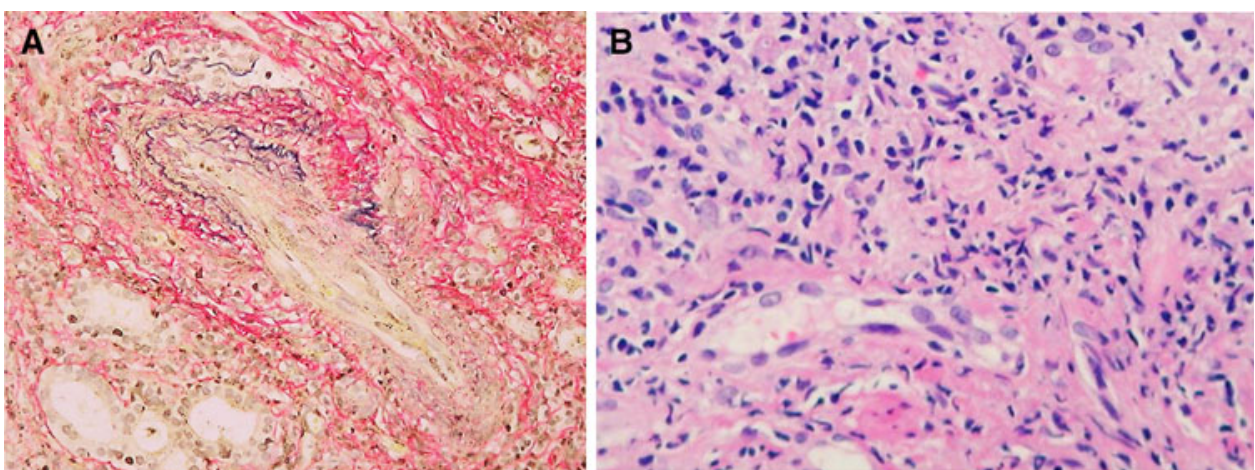

countries [1]. However, in Asian countries, various reports revealed that the ratio of MPO-ANCA-positive AAV is relatively higher [2-4]. We also checked the clinical features of GPA patients in our hospital since 1998, including the positivity rate of ANCA. Out of a total of 29 GPA patients, 9 patients $(31.0 \%)$ were MPO-ANCA-positive. We also found that there is a tendency that the symptoms of MPO-ANCA GPA are slightly different from PR3-ANCA GPA. It has been thought that the progression of disease from E (eyes, ears, nose, and upper airway) to either EL (lung) or EK (kidney) and then to ELK is well described in PR3-ANCA-positive GPA. However, our data showed that there was a tendency for $\mathrm{K}$ to precede $\mathrm{L}$ or $\mathrm{E}$ in MPOANCA-positive GPA. We thought that this patient suffered MPO-ANCA-positive GPA from the onset, partly because kidney lesions precede saddle nose and it was compatible with the characteristic of MPO-ANCA-positive GPA.

\section{ANCA-negative relapse of AAV}

It is not rare that an AAV patient who has been in remission with negative ANCA relapses without any elevation of ANCA titer, as described in this case. We checked the transition of ANCA titer of $24 \mathrm{AAV}$ patients [18 MPA, two GPA, two eosinophilic granulomatosis with polyangiitis (EGPA)] in our hospital who relapsed with serial measurements of ANCA titer during remission. A total of 6 of 24 patients $(25 \%)$ relapsed without any elevation of the ANCA titer. Thus, we should be mindful for a relapse, even if the ANCA titer remains negative. We have also reported that most of the patients whose ANCA was not completely negative at remission induction relapsed with elevation of ANCA (8/10: $80 \%$ ) [5]. There was no specific tendency regarding clinical manifestations in these patients who relapsed with negative ANCA.

There have been several reports discussing the relationship between relapse and ANCA titer [6-11]. Most of them showed that a rise in ANCA titer was related to a relapse, but some of them showed only a limited predictive role of serial ANCA measurement. When ANCA was able to be detected only by IF, there was a report by Gaskin et al. [6]. In 19 patients, ANCA were detectable, and six of these relapsed. In 18 patients, ANCA were undetectable, and none of these patients relapsed. In 33 patients, ANCA were intermittently present, nine of these relapsed, and all had ANCA detectable at the time of relapse. In six of the nine cases, relapse was accompanied or closely preceded by the reappearance of ANCA. In 2000, a report was published by Boomsma et al. [7], in which they checked the ANCA titer every 2 months and investigated the relationship between ANCA titer and relapse. Relapses occurred in 37 of 100 GPA patients and $34(92 \%)$ of the 37 patients showed a rise in the level of ANCA preceding their relapse, as detected by ELISA or indirect immunofluorescence (IIF). The predictive value of an increase in ANCA titers for relapse was $57 \%$ (17 of 30) for c-ANCA by IF, $71 \%$ (27 of 38 ) for PR3-ANCA by ELISA, and $100 \%$ (3 of 3) for MPO-ANCA by ELISA. They concluded that the serial measurement of ANCA levels is valuable for the early prediction of relapses in patients with GPA.

On the other hand, Finkielman et al. [8] checked the ANCA levels and disease activity of 156 GPA patients throughout the United States. The ANCA levels were only weakly associated with disease activity across patients, and changes in the ANCA levels explained less than $10 \%$ of the variation in disease activity. The decreases in ANCA levels were not statistically significantly associated with shorter time to remission, and increases in ANCA levels were not associated with relapse.

Thus, based on the current case and the reports above, both a rise in ANCA and persistently positive ANCA are significantly associated with disease relapse, although serial ANCA testing is not sufficient for treatment decisions.

\section{Detection of ANCA by ELISA}

We hypothesized that there are two possibilities for ANCA-negative relapse of AAV. One possibility is that, 
although disease activity was increased at relapse, ANCA was not produced in parallel with disease activity. Another possibility is that ANCA was actually positive at the relapse; however, ANCA had been changed so as not to be detected by the same ELISA kit. In this regard, a method of ANCA measurement is very important.

There are several types of ELISA systems for ANCA detection, such as direct ELISA, capture ELISA, anchor ELISA, and other third-generation ELISAs. Capture ELISA and third-generation ELISAs are thought to have high specificity. However, it is possible that these ELISA systems do not have superiority in sensitivity over conventional ELISA [12]. Thus, it is important to change the detection system if clinical symptoms are worsened while ANCA is still negative.

There have been a few reports of antibody-negative relapse of anti-glomerular basement membrane (GBM)positive Goodpasture syndrome [13-15]. In these cases, the investigators explained that it was because only local antibodies were reactivated and this did not lead to systemic antibody production. In the current case, p-ANCA by IF was weakly positive at relapse. This suggested that it was possible that epitope specificity had been changed in the way that ELISA could not detect.

Conflict of interest All the authors have declared no competing interest.

\section{References}

1. Hagen EC, Daha MR, Hermans J, Andrassy K, Csernok E, Gaskin G, Lesavre P, Lüdemann J, Rasmussen N, Sinico RA, Wiik A, van der Woude FJ. Diagnostic value of standardized assays for anti-neutrophil cytoplasmic antibodies in idiopathic systemic vasculitis. EC/BCR Project for ANCA Assay Standardization. Kidney Int. 1998;53:743-53.

2. Yamagata K, Usui J, Saito C, Yamaguchi N, Hirayama K, Mase K, Kobayashi M, Koyama A, Sugiyama H, Nitta K, Wada T, Muso E, Arimura Y, Makino H, Matsuo S. ANCA-associated systemic vasculitis in Japan: clinical features and prognostic changes. Clin Exp Nephrol. 2012;16:580-8.

3. Ozaki S, Atsumi T, Hayashi T, Ishizu A, Kobayashi S, Kumagai S, Kurihara Y, Kurokawa MS, Makino H, Nagafuchi H, Nakabayashi K, Nishimoto N, Suka M, Tomino Y, Yamada H, Yamagata K, Yoshida M, Yumura W, Amano K, Arimura Y, Hatta K, Ito S, Kikuchi H, Muso E, Nakashima H, Ohsone Y, Suzuki Y, Hashimoto H, Koyama A, Matsuo S, Kato H. Severity-based treatment for Japanese patients with MPO-ANCA-associated vasculitis: the JMAAV study. Mod Rheumatol. 2012; 22:394-404.

4. Chen M, Yu F, Wang SX, Zou WZ, Zhang Y, Zhao MH, Wang HY. Renal histology in Chinese patients with anti-myeloperoxidase autoantibody-positive Wegener's granulomatosis. Nephrol Dial Transplant. 2007;22:139-45.

5. Arimura Y, Kawashima S, Yoshihara K. ANCA associated vasculitis and PRGN. Nihon Jinzo Gakkai Shi. 2009;51:88-93.

6. Gaskin G, Savage CO, Ryan JJ, Jones S, Rees AJ, Lockwood $\mathrm{CM}$, Pusey CD. Anti-neutrophil cytoplasmic antibodies and disease activity during long-term follow-up of 70 patients with systemic vasculitis. Nephrol Dial Transplant. 1991;6:689-94.

7. Boomsma MM, Stegeman CA, van der Leij MJ, Oost W, Hermans J, Kallenberg CG, Limburg PC, Tervaert JW. Prediction of relapses in Wegener's granulomatosis by measurement of antineutrophil cytoplasmic antibody levels: a prospective study. Arthritis Rheum. 2000;43:2025-33.

8. Finkielman JD, Merkel PA, Schroeder D, Hoffman GS, Spiera R, St Clair EW, Davis JC Jr, McCune WJ, Lears AK, Ytterberg SR, Hummel AM, Viss MA, Peikert T, Stone JH, Specks U; WGET Research Group. Antiproteinase 3 antineutrophil cytoplasmic antibodies and disease activity in Wegener granulomatosis. Ann Intern Med. 2007;147:611-9.

9. Han WK, Choi HK, Roth RM, McCluskey RT, Niles JL. Serial ANCA titers: useful tool for prevention of relapses in ANCAassociated vasculitis. Kidney Int. 2003;63:1079-85.

10. Damoiseaux J, Dähnrich C, Rosemann A, Probst C, Komorowski L, Stegeman CA, Egerer K, Hiepe F, van Paassen P, Stöcker W, Schlumberger W, Tervaert JW. A novel enzyme-linked immunosorbent assay using a mixture of human native and recombinant proteinase- 3 significantly improves the diagnostic potential for antineutrophil cytoplasmic antibody-associated vasculitis. Ann Rheum Dis. 2009;68:228-33.

11. Terrier B, Saadoun D, Sène D, Ghillani P, Amoura Z, Deray G, Fautrel B, Piette JC, Cacoub P. Antimyeloperoxidase antibodies are a useful marker of disease activity in antineutrophil cytoplasmic antibody-associated vasculitides. Ann Rheum Dis. 2009;68:1564-71.

12. Ito-Ihara T, Muso E, Kobayashi S, Uno K, Tamura N, Yamanishi Y, Fukatsu A, Watts RA, Scott DG, Jayne DR, Suzuki K, Hashimoto $\mathrm{H}$. A comparative study of the diagnostic accuracy of ELISA systems for the detection of anti-neutrophil cytoplasm antibodies available in Japan and Europe. Clin Exp Rheumatol. 2008;26:1027-33.

13. Klasa RJ, Abboud RT, Ballon HS, Grossman L. Goodpasture's syndrome: recurrence after a five-year remission. Case report and review of the literature. Am J Med. 1988;84:751-5.

14. Salama AD, Dougan T, Levy JB, Cook HT, Morgan SH, Naudeer S, Maidment G, George AJ, Evans D, Lightstone L, Pusey CD. Goodpasture's disease in the absence of circulating anti-glomerular basement membrane antibodies as detected by standard techniques. Am J Kidney Dis. 2002;39:1162-7.

15. Benz K, Amann K, Dittrich K, Hugo C, Schnur K, Dötsch J. Patient with antibody-negative relapse of Goodpasture syndrome. Clin Nephrol. 2007;67:240-4. 